\title{
Research on the Coordination Level Evaluation of Technology, Economy, Society and Ecology Coupling in Beijing-Tianjin-Hebei Region
}

\author{
Dan $\mathrm{Wu}^{1, *}$ Siqi $\mathrm{Cao}^{1}$ \\ ${ }^{1}$ School of Economics and Management, North China University of Technology, Beijing 100144, China \\ *Corresponding author.Email:wu_daniel@163.com
}

\begin{abstract}
From the four dimensions of technology, economy, society, and ecology, this paper adopts the policy literature review method, combining the two categories of "policy orientation" and "document reference" to conduct qualitative screening of evaluation indicators for the coordination level of technology-economy-society-ecological coupling in the Beijing-Tianjin-Hebei region. On this basis, it uses the principal component-correlation analysis method to quantitatively screen the evaluation indicators of the coordination level of technology-economy-society-ecology coupling in the BeijingTianjin-Hebei region. At the same time, it combines the weighted comprehensive index method with the coupling coordination model to construct a technology-economy-society-ecology coupling coordination evaluation model to measure the technology-economy-society-ecology development index of the BeijingTianjin-Hebei region and dynamically evaluate the coordination level of technology-economy-societyecology coupling in the Beijing-Tianjin-Hebei region.
\end{abstract}

Keywords: Beijing-Tianjin-Hebei region, technology-economy-society-ecology, coupling, coordination level,

evaluation

\section{INTRODUCTION}

The Fifth Plenary Session of the 18th CPC National Congress put forward the five development concepts of "innovation, coordination, green, openness, and sharing", which provided important strategic support for achieving national development goals during the "13th Five-Year Plan" period, solving problems in national economic and social development, and strengthening development advantages. The report of the 19th National Congress of the Communist Party of China put forward "implementing the new development concept and building a modern economic system", and emphasized the need to accelerate the construction of an industrial system with the coordinated development of technology, real economy, modern finance, and human resources. Therefore, improving scientific and technological innovation capabilities is an inexhaustible driving force for the sustainable economic and social development of the Beijing-Tianjin-Hebei region. The implementation of the concept of green development is to accelerate the formation of a new pattern of ecological and environmental changes in the harmonious development of man and nature in the

*Project: Beijing Social Science Foundation Youth Project (17GLC064).
Beijing-Tianjin-Hebei region. And leading the transformation and upgrading of the real economy in the Beijing-Tianjin-Hebei region with technological innovation, consolidating the foundation for sustainable development with green development, and promoting the coordinated coupling development of technology, economy, society, and ecology are the focus of the sustainable economic and social development of the Beijing-Tianjin-Hebei region. To this end, a comprehensive evaluation of the coordination level of technology-economy-society-ecology coupling in the Beijing-Tianjin-Hebei region is conducted.

\section{LITERATURE REVIEW}

The coordination evaluation between regional science and technology, economic and social development, and ecological environment construction mainly includes three aspects: coordination evaluation between technology and economic development [1-13], coordination evaluation between technology and ecological environment construction [14] [15] [16], and evaluation of the coordination between science and technology, economic development, and ecological environment construction [17] [18] [19] [20]. With reference to the existing literature, due to the ease of quantification, the scientific and technological 
indicators, scientific and technological financial investment and scientific and technological human investment have been widely adopted as scientific and technological investment indicators, such as scientific and technological activity expenditure, R\&D investment intensity, internal $\mathrm{R} \& \mathrm{D}$ expenditure, scientific and technological activity personnel, R\&D personnel full-time equivalent and so on. Science and technology output indicators are divided into direct output indicators and indirect output indicators. The direct output indicators are mainly papers and patents, and the indirect output indicators are reflected in the marketization of scientific research results, such as the contract value of the technology market, the output of new products, and the output value of high-tech output. For economic and social development indicators, it focuses on the level of economic development, industrial structure, national income, education level, employment status, and urbanization, such as GDP, the proportion of the output value of the tertiary industry, Engel coefficient, education expenditure level, unemployment rate, the proportion of urban population, etc. Aiming at the ecological environment construction indicators, it focuses on the pollutant discharge, pollution control, and resource and energy stocks, such as wastewater and waste discharge, pollution control investment, waste treatment and utilization, forest coverage, water resources utilization, cultivated land area, etc.

With reference to the existing literature, in the design of the evaluation index system, scholars focus on qualitative analysis, and screen the index based on the importance of the index and the availability of index data, which is highly subjective. To this end, it adopts the policy literature review method from the four dimensions of technology, economy, society, and ecology, combines the two categories of "policy orientation" and "document reference", and conducts qualitative screening of evaluation indicators for the coordination level of technology-economy-societyecology coupling in the Beijing-Tianjin-Hebei region. On this basis, it uses the principal componentcorrelation analysis method to quantitatively screen the evaluation indicators of the coordination level of technology-economy-society-ecology coupling. At the same time, it combines the weighted comprehensive index method with the coupling coordination model to construct a technology-economy-society-ecology coupling coordination evaluation model to measure the technology-economy-society-ecology development index of the Beijing-Tianjin-Hebei region and dynamically evaluate the coordination level of technology-economy-society-ecology coupling in the Beijing-Tianjin-Hebei region.

\section{THE INDEX DESIGN OF THE COORDINATION LEVEL EVALUATION OF TECHNOLOGY-ECONOMY-SOCIETY-ECOLOGY COUPLING IN THE BEIJING-TIANJIN-HEBEI REGION}

It refers to the "Beijing-Tianjin-Hebei Coordinated Development Plan" and "Beijing-Tianjin-Hebei National Economic and Social Development Plan During the 13th Five-Year Plan" and other policy documents to clarify the "policy-oriented" indicators for the development of the Beijing-Tianjin-Hebei region. At the same time, it refers to the core journal literature with the theme of "Technology, Economy, Society, and Ecology of Beijing-Tianjin-Hebei", and adopts the literature review method to determine the "document reference" indicators for the development of the Beijing-Tianjin-Hebei region. As the Beijing-TianjinHebei region involves many related indicators in technology, economy, society and ecology, it uses the principal component-correlation analysis method to screen the indicators for dimensionality reduction. First, it uses principal component analysis to quantitatively pre-screen the initial indicators according to the factor loading. It screens the index whose principal component factor load is greater than 0.9 and the absolute value of the second or third principal component factor load is the largest. Secondly, it uses the correlation analysis method to perform a secondary quantitative screening of the indicators left after the principal component analysis screening according to the size of the correlation coefficient. It respectively calculates the correlation coefficient between any two indicators under the secondary indicator level in the dimensions of technology, economy, society and ecology in the Beijing-Tianjin-Hebei region, and sets the threshold value $M(0<M<1)$ of the index correlation coefficient. If the correlation coefficient between the two indicators is less than the threshold M, then both indicators are retained at the same time; if the correlation coefficient between the two indicators is greater than the threshold $M$, then it deletes the indicator with the smaller absolute value of the factor load in the two indicators, that is, the indicator with very little influence on the evaluation result. In this paper, the threshold $\mathrm{M}=0.9$. The Beijing-TianjinHebei region's technology-economy-society-ecology coupling coordination level evaluation index system is divided into three levels: dimension level, criterion level and index level, including 4 dimensions and objectives, 16 criteria, and 28 indicators (see "Table I"). 
TABLE I. THE BEIJING-TIANJIN-HEBEI REGION'S TECHNOLOGY-ECONOMY-SOCIETY-ECOLOGY COUPLING COORDINATION LEVEL EVALUATION INDEX SYSTEM

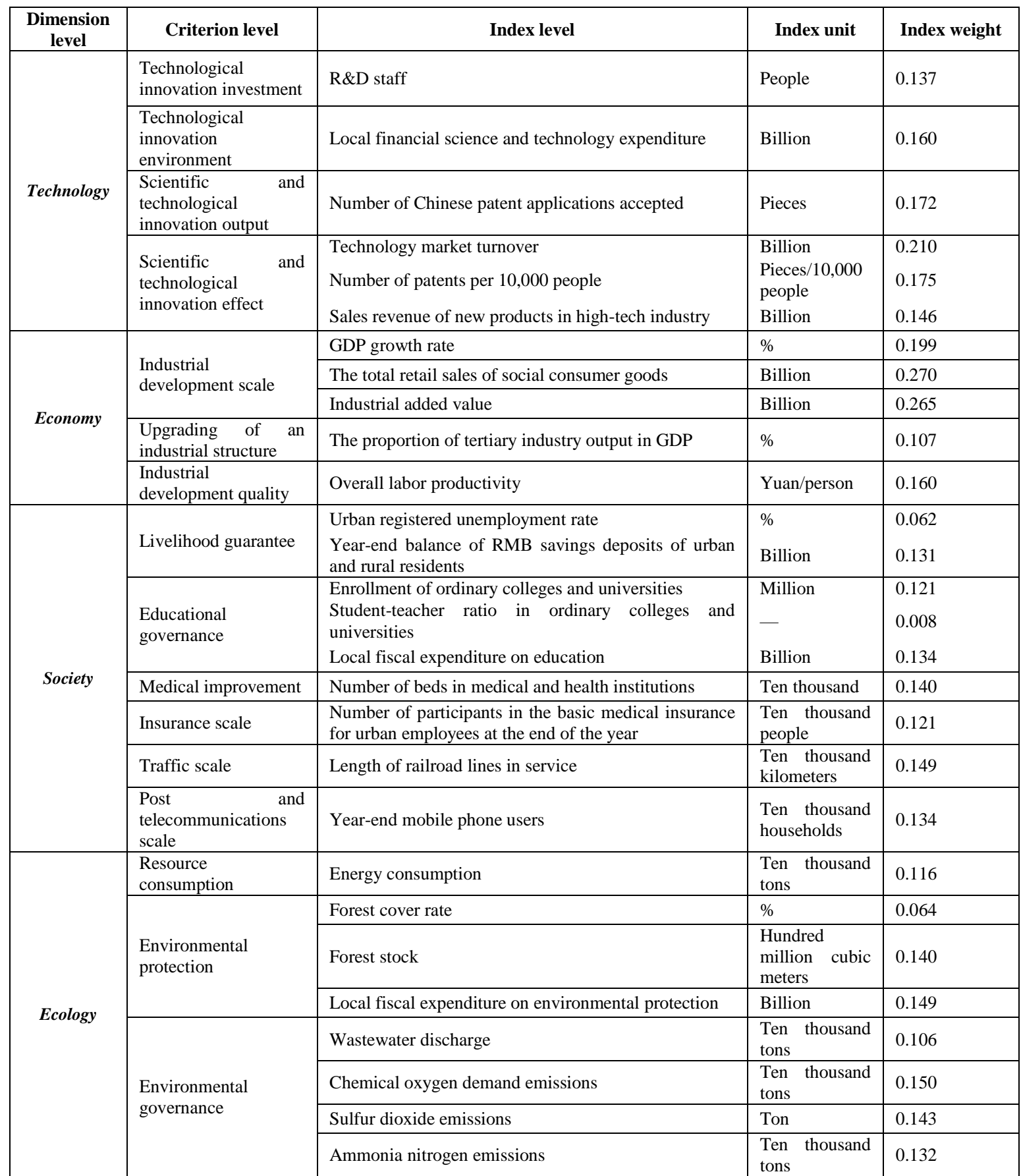

In "Table I", the index weights of different dimensions are determined by the coefficient of variation method, which can be expressed as: 


$$
\left\{\begin{array}{l}
w_{k i}=\frac{V_{k i}}{\sum_{i=1}^{m} V_{k i}} \\
V_{k i}=\frac{\sigma_{k i}}{\frac{1}{n} \sum_{i=1}^{n} x_{k i}}
\end{array}\right.
$$

In formula (1), $w_{k i}$ is the weight of the $k$ dimension and the $i$ indicator $(k=1, k=2, k=3$, and $k=4$ represent the 4 dimensions of technology, economy, society, and ecology respectively), and $V_{k i}$ is the coefficient of variation of the $i$ index of the $k$ dimension. $\sum_{i=1}^{m} V_{k i}$ is the sum of the variable coefficients

$$
\begin{gathered}
W_{j k}(t)=\sum_{i=1}^{m}\left[w_{k i} \cdot x_{j k i}(t)\right] \\
x_{j k i}(t)=\left\{\begin{array}{l}
\frac{a_{j k i}(t)-\min a_{j k i}(t)}{\max a_{j k i}(t)-\min a_{j k i}(t)} \\
\frac{\max a_{j k i}(t)-a_{j k i}(t)}{\max a_{j k i}(t)-\min a_{j k i}(t)}
\end{array} \quad a_{j k i}\right. \text { is cost index }
\end{gathered}
$$

In formula (2), $W_{j k}(t)$ is the development index of the ${ }^{k}$ dimension of the ${ }^{j}$ area in the ${ }^{t}$ period, ${ }^{x_{j k i}}(t)$ is the dimensionless index value, ${ }^{a_{j k i}(t)}$ is the ${ }^{i}$ index value of the $k$ dimension of the ${ }^{j}$ region in the ${ }^{t}$ period, and $\max a_{j k i}(t)$ and $\min a_{j k i}(t)$ are respectively the best value and the worst value of the $i$ index value of the ${ }^{k}$ dimension in the ${ }^{j}$ area in the ${ }^{t}$ period.

Based on the weighted comprehensive index method, it constructs a coupled and coordinated development model to dynamically evaluate the technology-economy-society-ecology coupling and coordination level of the Beijing-Tianjin-Hebei region, which can be expressed as:

$$
\begin{aligned}
& D_{j}(t)=\sqrt{C_{j}(t) \cdot P_{j}(t)} \\
& \left\{\begin{array}{l}
C_{j}(t)=\left[\frac{\prod_{k=1}^{4} W_{j k}(t)}{P_{j}(t)^{k}}\right]^{\frac{1}{k}} \\
P_{j}(t)=\frac{1}{k} \sum_{k=1}^{4} W_{j k}(t)
\end{array}\right.
\end{aligned}
$$

of the indicators of the $k$ dimension, $\sigma_{k i}$ is the standard deviation of the $i$ index of the $k$ dimension, and $\frac{1}{n} \sum_{i=1}^{n} x_{k i}$ is the mean value of the $i$ index of the $k$ dimension.

\section{THE BEIJING-TIANJIN-HEBEI REGION'S TECHNOLOGY-ECONOMY-SOCIETY-ECOLOGY COUPLING COORDINATION LEVEL EVALUATION}

\section{A. Evaluation model construction}

It adopts the weighted comprehensive index method to construct the scientific and technological, economic, social and ecological development index model of the Beijing-Tianjin-Hebei region, which can be expressed as:

In formula (3), $D_{j}(t)$ and $(j=1,2,3,4)$ is the coupling coordination level of technology-economysociety-ecology in the $j$ area during the ${ }^{t}$ period, where $j=1, j=2, j=3$, and $j=4$ are respectively Beijing, Tianjin, Hebei, and Beijing-Tianjin-Hebei as a whole. $C_{j}(t)$ is the coupling index of technologyeconomy-society-ecology in area $j$ during period $t$, which measures the degree of coupling correlation between technological innovation and economic development, social governance, and ecological construction. $P_{j}(t)$ is the coordination index of technology-economy-society-ecology in area ${ }^{j}$ during period ${ }^{t}$, which measures the degree of coordination between technological innovation and economic development, social governance, and ecological construction.

According to formula (1)-formula (3), the relative development model is used to measure the relative development level of technology, economy, society and ecology in the Beijing-Tianjin-Hebei region, which can be expressed as:

$$
E_{j k k^{\prime}}(t)=\frac{W_{j k}(t)}{W_{j k^{\prime}}(t)}
$$


In formula (4), ${ }_{j k k^{\prime}}(t)$ is the relative development level of the $k$ and $k^{\prime}$ dimensions of the $j$ area during the ${ }^{t}$ period, $k=1$ is the technological dimension, and $k^{\prime}=2, k^{\prime}=3$, and $k^{\prime}=4$ are the economic, social, and ecological dimensions respectively.
According to formula (1)-formula (4), according to the coupling coordination level and relative development level, it refers to the existing literature classification results to determine the coordination level and stage of technology-economy-society-ecology coupling in the Beijing-Tianjin-Hebei region, see "Table II".

TABLE II. COORDINATION LEVELS AND STAGES OF TECHNOLOGY-ECONOMY-SOCIETY-ECOLOGY COUPLING IN THE BEIJING-TIANJIN-HEBEI REGION

\begin{tabular}{|c|c|c|c|c|c|}
\hline \multirow{2}{*}{ Interval } & \multirow{2}{*}{$\begin{array}{l}\text { Coupling } \\
\text { coordination } \\
\text { level }\end{array}$} & \multirow{2}{*}{$\begin{array}{l}\text { Coupling } \\
\text { coordination } \\
\text { level }\end{array}$} & \multirow{2}{*}{$\begin{array}{l}\text { Relative } \\
\text { development } \\
\text { level }\end{array}$} & \multicolumn{2}{|c|}{ Coupling coordination development stage } \\
\hline & & & & Stage characteristics & Stage decision \\
\hline \multirow{4}{*}{$0<\mathrm{D} \leq 0.4$} & $(0,0.1]$ & $\begin{array}{l}\text { Extreme } \\
\text { imbalance }\end{array}$ & $0<\mathrm{E}<0.8$ & $\begin{array}{l}\text { Technology lags behind and } \\
\text { restricts the economy } \\
\begin{array}{l}\text { Development } \\
\text { governance }\end{array} \text { (social } \\
\text { construction) }\end{array}$ & $\begin{array}{l}\text { System tends to } \\
\text { degenerate }\end{array}$ \\
\hline & $(0.1,0.2]$ & $\begin{array}{l}\text { Serious } \\
\text { imbalance }\end{array}$ & \multirow[b]{2}{*}{$0.8 \leq \mathrm{E} \leq 1.2$} & \multirow{2}{*}{$\begin{array}{l}\text { Synchronize technology and } \\
\text { promote economy } \\
\begin{array}{l}\text { Development } \\
\text { governance or } \\
\text { construction) }\end{array}\end{array}$} & \multirow{2}{*}{$\begin{array}{l}\text { System tends to be } \\
\text { optimized }\end{array}$} \\
\hline & $(0.2,0.3]$ & $\begin{array}{l}\text { Moderate } \\
\text { imbalance }\end{array}$ & & & \\
\hline & $(0.3,0.4]$ & Mild imbalance & $\mathrm{E}>1.2$ & $\begin{array}{lrr}\text { Technology advances } & \text { and } \\
\text { affects the economy } & \\
\text { Development } & & \text { (social } \\
\text { governance } & \text { or } & \text { ecological } \\
\text { construction) } & & \end{array}$ & $\begin{array}{l}\text { System tends to } \\
\text { degenerate }\end{array}$ \\
\hline \multirow{3}{*}{$0.4<\mathrm{D} \leq 0.6$} & $(0.4,0.5]$ & $\begin{array}{l}\text { On the verge of } \\
\text { imbalance }\end{array}$ & $0<\mathrm{E}<0.8$ & $\begin{array}{l}\text { Technology lags behind and } \\
\text { restricts the economy } \\
\begin{array}{l}\text { Development } \\
\text { governance or } \\
\text { construction) }\end{array}\end{array}$ & $\begin{array}{l}\text { System tends to } \\
\text { degenerate }\end{array}$ \\
\hline & \multirow{2}{*}{$(0.5,0.6]$} & \multirow{2}{*}{$\begin{array}{l}\text { Barely } \\
\text { coordinated }\end{array}$} & $0.8 \leq \mathrm{E} \leq 1.2$ & 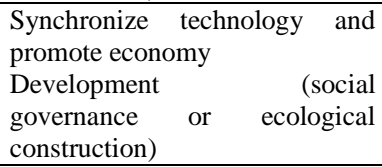 & $\begin{array}{l}\text { System tends to be } \\
\text { optimized }\end{array}$ \\
\hline & & & $\mathrm{E}>1.2$ & $\begin{array}{lrr}\text { Technology advances } & \text { and } \\
\text { affects the economy } & \\
\text { Development } & & \text { (social } \\
\text { governance } & \text { or } & \text { ecological } \\
\text { construction) } & & \end{array}$ & $\begin{array}{l}\text { System tends to } \\
\text { degenerate }\end{array}$ \\
\hline \multirow{4}{*}{$0.6<\mathrm{D} \leq 1$} & $(0.6,0.7]$ & $\begin{array}{l}\text { Primary } \\
\text { coordination }\end{array}$ & $0<\mathrm{E}<0.8$ & $\begin{array}{l}\text { Technology lags behind and } \\
\text { restricts the economy } \\
\begin{array}{l}\text { Development } \\
\text { governance }\end{array} \text { (social } \\
\text { construction) }\end{array}$ & $\begin{array}{l}\text { System tends to } \\
\text { degenerate }\end{array}$ \\
\hline & $(0.7,0.8]$ & $\begin{array}{l}\text { Moderate } \\
\text { coordination }\end{array}$ & \multirow[b]{2}{*}{$0.8 \leq \mathrm{E} \leq 1.2$} & \multirow{2}{*}{$\begin{array}{l}\text { Synchronize technology and } \\
\text { promote economy } \\
\begin{array}{l}\text { Development } \\
\text { governance }\end{array} \\
\begin{array}{l}\text { construction) } \\
\text { or }\end{array}\end{array}$} & \multirow{2}{*}{$\begin{array}{l}\text { System tends to be } \\
\text { optimized }\end{array}$} \\
\hline & $(0.8,0.9]$ & $\begin{array}{l}\text { Good } \\
\text { coordination }\end{array}$ & & & \\
\hline & $(0.9,1]$ & $\begin{array}{l}\text { High-quality } \\
\text { coordination }\end{array}$ & $\mathrm{E}>1.2$ & $\begin{array}{lrr}\begin{array}{l}\text { Technology advances } \\
\text { affects the economy }\end{array} & \text { and } \\
\text { Development } & & \text { (social } \\
\text { governance } & \text { or } & \text { ecological } \\
\text { construction) } & & \\
\end{array}$ & $\begin{array}{l}\text { System tends to } \\
\text { degenerate }\end{array}$ \\
\hline
\end{tabular}

a. Note: "Table II" is designed by the author according to reference [21].

\section{B. Analysis of evaluation results}

1) Evaluation of the technology-economy coupling coordination level: According to the established index system, coupling coordination model and relative development model, it substitutes the scientific and technological innovation and economic development level index into the calculation to obtain the calculation 
result of the coordinated development level of technology and economy (see "Table III").

It can be seen from "Table III": First, from 2010 to 2017, the degree of coupling coordination of the Beijing-Tianjin-Hebei technology-economy system increased from 0.41 to 0.94 , and the level of coupling coordination transitioned from "on the verge of imbalance" to "high-quality coordination". From the perspective of the trend of the coupling coordination development level, the coordinated development of technology-economy coupling in Beijing and Tianjin from 2010 to 2013 was faster, and the degree of coupling coordination increased from 0.44 and 0.4 to 0.71 and 0.77 respectively, with an average annual growth rate of 0.09 and 0.12 ; Beijing and Tianjin slowed down the coordinated development of technology and economy coupling from 2014 to 2017, and the degree of coupling coordination increased from 0.77 and 0.83 to 0.95 and 0.9 respectively, with an average annual growth rate of 0.06 and 0.02 . From 2010 to 2012, Hebei had a faster coordinated development of technology and economy coupling, with the coupling coordination degree increasing from 0.33 to 0.63 , with an average annual growth rate of 0.1 ; Hebei slowed down the coordinated development of technology and economy coupling from 2013 to 2017, and the coupling coordination degree increased from 0.66 to 0.96 , with an average annual growth rate of 0.08 . From the perspective of the level of coupling coordination development, Beijing and Tianjin have entered the stage of "high-quality coordination" development of technology and economy earlier than Hebei.

Second, from 2010 to 2017, the relative development of the Beijing-Tianjin-Hebei technologyeconomy system generally went through the "lagging behind-synchronizing" stage. The technological innovation has changed from restricting economic development to promoting economic development, and the system tends to be optimized. Among them, Beijing had been in the stage of lagging technological innovation and restricting economic development from 2010 to 2012, and in 2013 and 2015-2017 at the stage of synchronizing technological innovation and promoting economic development. Only in 2014, its technological innovation was slightly ahead of economic development. Tianjin had been in the stage of lagging technological innovation and restricting economic development from 2010 to 2012. It was in a stage of synchronizing technological innovation and promoting economic development in 2013-2014 and 2016-2017. Only in 2015, its technological innovation was slightly ahead of economic development. Hebei had been in the stage of lagging technological innovation and restricting economic development from 2010 to 2014. From 2015 to 2017, it was in a stage of synchronizing technological innovation and promoting economic development.

TABLE III. MEASUREMENT RESULTS OF THE COORDINATED DEVELOPMENT LEVEL OF TECHNOLOGY-ECONOMY-SOCIETY-ECOLOGY COUPLING IN THE BEIJING-TIANJIN-HEBEI REGION

\begin{tabular}{|c|c|c|c|c|c|c|c|c|c|c|c|c|c|c|c|}
\hline \multirow{2}{*}{ Area } & \multirow{2}{*}{ Year } & \multicolumn{4}{|c|}{ Technology-economy } & \multicolumn{4}{|c|}{ Technology-society } & \multicolumn{4}{|c|}{ Technology-ecology } & \multicolumn{2}{|c|}{\begin{tabular}{|c|}
$\begin{array}{c}\text { Technology- } \\
\text { economy-society- } \\
\text { ecology }\end{array}$ \\
\end{tabular}} \\
\hline & & D & Level & $\mathbf{E}$ & Stage & D & Level & $\mathbf{E}$ & Stage & D & Level & $\mathbf{E}$ & Stage & D & Level \\
\hline \multirow{8}{*}{ Beijing } & 2010 & 0.44 & $\begin{array}{|lr|}\text { On r r } & \text { the } \\
\text { verge } & \text { of } \\
\text { imbalance }\end{array}$ & 0.36 & $\begin{array}{l}\text { Lagging } \\
\text { behind }\end{array}$ & 0.37 & Mild imbalance & 0.75 & $\begin{array}{l}\text { Lagging } \\
\text { behind }\end{array}$ & 0.47 & $\begin{array}{l}\text { On the verge of } \\
\text { imbalance }\end{array}$ & $\mathrm{f} \mid 0.30$ & $\begin{array}{l}\text { Lagging } \\
\text { behind }\end{array}$ & 0.47 & $\begin{array}{l}\text { On the verge } \\
\text { of imbalance }\end{array}$ \\
\hline & 2011 & 0.57 & \begin{tabular}{|l|} 
Barely \\
coordinated
\end{tabular} & 0.57 & $\begin{array}{l}\text { Lagging } \\
\text { behind }\end{array}$ & 0.51 & $\begin{array}{l}\text { Barely } \\
\text { coordinated }\end{array}$ & 0.87 & Synchronizing & 0.46 & $\begin{array}{l}\text { On the verge of } \\
\text { imbalance }\end{array}$ & f 1.29 & Ahead of & 0.52 & $\begin{array}{l}\text { Barely } \\
\text { coordinated }\end{array}$ \\
\hline & 2012 & 0.59 & \begin{tabular}{|l|} 
Barely \\
coordinated
\end{tabular} & 0.76 & $\begin{array}{l}\text { Lagging } \\
\text { behind }\end{array}$ & 0.65 & $\begin{array}{l}\text { Primary } \\
\text { coordination }\end{array}$ & 0.51 & $\begin{array}{l}\text { Lagging } \\
\text { behind }\end{array}$ & 0.51 & $\begin{array}{l}\text { Barely } \\
\text { coordinated }\end{array}$ & 1.41 & Ahead of & 0.60 & $\begin{array}{l}\text { Barely } \\
\text { coordinated }\end{array}$ \\
\hline & 2013 & 0.71 & $\begin{array}{l}\text { Moderate } \\
\text { coordination }\end{array}$ & 0.97 & Synchronizing & 0.78 & $\begin{array}{l}\text { Moderate } \\
\text { coordination }\end{array}$ & 0.70 & $\begin{array}{l}\text { Lagging } \\
\text { behind }\end{array}$ & 0.58 & $\begin{array}{l}\text { Barely } \\
\text { coordinated }\end{array}$ & 2.27 & Ahead of & 0.67 & $\begin{array}{l}\text { Primary } \\
\text { coordination }\end{array}$ \\
\hline & 2014 & 0.77 & \begin{tabular}{|l|} 
Moderate \\
coordination
\end{tabular} & 1.29 & Ahead of & 0.85 & $\begin{array}{l}\text { Good } \\
\text { coordination }\end{array}$ & 0.90 & Synchronizing & 0.64 & $\begin{array}{l}\text { Primary } \\
\text { coordination }\end{array}$ & 2.72 & Ahead of & 0.71 & $\begin{array}{l}\text { Moderate } \\
\text { coordination }\end{array}$ \\
\hline & 2015 & 0.81 & $\left|\begin{array}{l}\text { Good } \\
\text { coordination }\end{array}\right|$ & 1.15 & Synchronizing & 0.84 & $\begin{array}{l}\text { Good } \\
\text { coordination }\end{array}$ & 0.98 & Synchronizing & 0.69 & $\begin{array}{l}\text { Primary } \\
\text { coordination }\end{array}$ & 2.19 & Ahead of & 0.75 & $\begin{array}{l}\text { Moderate } \\
\text { coordination }\end{array}$ \\
\hline & 2016 & 0.91 & \begin{tabular}{|l|} 
High- \\
quality \\
coordination
\end{tabular} & 1.06 & Synchronizing & 0.90 & $\begin{array}{l}\text { Good } \\
\text { coordination }\end{array}$ & 1.12 & Synchronizing & 0.82 & $\begin{array}{l}\text { Good } \\
\text { coordination }\end{array}$ & 1.60 & Ahead of & 0.85 & $\begin{array}{l}\text { Good } \\
\text { coordination }\end{array}$ \\
\hline & 2017 & 0.95 & \begin{tabular}{|l|}
$\begin{array}{l}\text { High- } \\
\text { quality } \\
\text { coordination }\end{array}$ \\
\end{tabular} & 1.16 & Synchronizing & 0.93 & $\begin{array}{l}\text { High-quality } \\
\text { coordination }\end{array}$ & 1.23 & Ahead of & 0.90 & $\begin{array}{l}\text { Good } \\
\text { coordination }\end{array}$ & 1.43 & Ahead of & 0.90 & $\begin{array}{l}\text { Good } \\
\text { coordination }\end{array}$ \\
\hline \multirow{4}{*}{ Tianjin } & 2010 & 0.40 & \begin{tabular}{|l} 
Mild \\
imbalance
\end{tabular} & 0.24 & $\begin{array}{l}\text { Lagging } \\
\text { behind }\end{array}$ & 0.34 & Mild imbalance & 0.47 & $\begin{array}{l}\text { Lagging } \\
\text { behind }\end{array}$ & 0.41 & $\begin{array}{l}\text { On the verge of } \\
\text { imbalance }\end{array}$ & $\mathrm{f} \mid 0.22$ & $\begin{array}{l}\text { Lagging } \\
\text { behind }\end{array}$ & 0.44 & $\begin{array}{l}\text { On the verge } \\
\text { of imbalance }\end{array}$ \\
\hline & 2011 & 0.56 & \begin{tabular}{|l|} 
Barely \\
coordinated
\end{tabular} & 0.41 & $\begin{array}{l}\text { Lagging } \\
\text { behind }\end{array}$ & 0.51 & $\begin{array}{l}\text { Barely } \\
\text { coordinated }\end{array}$ & 0.58 & $\begin{array}{l}\text { Lagging } \\
\text { behind }\end{array}$ & 0.43 & $\begin{array}{l}\text { On the verge of } \\
\text { imbalance }\end{array}$ & $\mathrm{f} \mid 1.16$ & Synchronizing & 0.52 & $\begin{array}{l}\text { Barely } \\
\text { coordinated }\end{array}$ \\
\hline & 2012 & 0.66 & $\begin{array}{l}\text { Primary } \\
\text { coordination }\end{array}$ & 0.70 & $\begin{array}{l}\text { Lagging } \\
\text { behind }\end{array}$ & 0.65 & $\begin{array}{l}\text { Primary } \\
\text { coordination }\end{array}$ & 0.74 & $\begin{array}{l}\text { Lagging } \\
\text { behind }\end{array}$ & 0.47 & $\begin{array}{l}\text { On the verge of } \\
\text { imbalance }\end{array}$ & f 2.74 & Ahead of & 0.58 & $\begin{array}{l}\text { Barely } \\
\text { coordinated }\end{array}$ \\
\hline & 2013 & 0.77 & $\begin{array}{l}\text { Moderate } \\
\text { coordination }\end{array}$ & 0.96 & Synchronizing & 0.79 & $\begin{array}{l}\text { Moderate } \\
\text { coordination }\end{array}$ & 0.89 & Synchronizing & 0.54 & $\begin{array}{l}\text { Barely } \\
\text { coordinated }\end{array}$ & 4.12 & Ahead of & 0.66 & $\begin{array}{l}\text { Primary } \\
\text { coordination }\end{array}$ \\
\hline
\end{tabular}




\begin{tabular}{|c|c|c|c|c|c|c|c|c|c|c|c|c|c|c|c|}
\hline & \multirow{2}{*}{ Year } & \multicolumn{4}{|c|}{ Technology-economy } & \multicolumn{4}{|c|}{ Technology-society } & \multicolumn{4}{|c|}{ Technology-ecology } & \multicolumn{2}{|c|}{$\begin{array}{c}\text { Technology- } \\
\text { economy-society- } \\
\text { ecology }\end{array}$} \\
\hline & & $\mathbf{D}$ & Level & $\mathbf{E}$ & Stage & D & Level & $\mathbf{E}$ & Stage & D & Level & $\mathbf{E}$ & Stage & D & Level \\
\hline & 2014 & 0.83 & \begin{tabular}{|l|} 
Good \\
coordination
\end{tabular} & 1.08 & Synchronizing 0 & 0.87 & $\begin{array}{l}\text { Good } \\
\text { coordination }\end{array}$ & 0.88 & Synchronizing 0 & 0.57 & $\begin{array}{l}\text { Barely } \\
\text { coordinated }\end{array}$ & 4.84 & Ahead of & 0.70 & \begin{tabular}{|l|} 
Primary \\
coordination
\end{tabular} \\
\hline & 2015 & 0.88 & $\begin{array}{l}\text { Good } \\
\text { coordination }\end{array}$ & 1.28 & Ahead of & 0.93 & $\begin{array}{l}\text { High-quality } \\
\text { coordination }\end{array}$ & 1.03 & Synchronizing 0 & 0.63 & $\begin{array}{l}\text { Primary } \\
\text { coordination }\end{array}$ & 4.76 & Ahead of & 0.74 & $\begin{array}{l}\text { Moderate } \\
\text { coordination }\end{array}$ \\
\hline & 2016 & 0.93 & \begin{tabular}{|l|} 
High- \\
quality \\
coordination
\end{tabular} & 1.19 & Synchronizing 0 & 0.97 & $\begin{array}{l}\text { High-quality } \\
\text { coordination }\end{array}$ & 1.03 & Synchronizing 0 & 0.81 & $\begin{array}{l}\text { Good } \\
\text { coordination }\end{array}$ & 2.08 & Ahead of & 0.86 & $\begin{array}{l}\text { Good } \\
\text { coordination }\end{array}$ \\
\hline & 2017 & 0.90 & \begin{tabular}{|l|} 
Good \\
coordination
\end{tabular} & 1.07 & Synchronizing 0 & 0.95 & $\begin{array}{l}\text { High-quality } \\
\text { coordination }\end{array}$ & 0.88 & Synchronizing 0 & 0.83 & $\begin{array}{l}\text { Good } \\
\text { coordination }\end{array}$ & 1.47 & Ahead of & 0.88 & \begin{tabular}{|l|} 
Good \\
coordination
\end{tabular} \\
\hline \multirow{8}{*}{ Hebei } & 2010 & 0.33 & $\begin{array}{l}\text { Mild } \\
\text { imbalance }\end{array}$ & 0.12 & $\begin{array}{l}\text { Lagging } \\
\text { behind }\end{array}$ & 0.27 & $\begin{array}{l}\text { Moderate } \\
\text { imbalance }\end{array}$ & 0.25 & $\begin{array}{l}\text { Lagging } \\
\text { behind }\end{array}$ & 0.36 & Mild imbalance & 0.08 & $\begin{array}{l}\text { Lagging } \\
\text { behind }\end{array}$ & 0.41 & $\begin{array}{l}\text { On the verge } \\
\text { of imbalance }\end{array}$ \\
\hline & 2011 & 0.53 & $\left|\begin{array}{l}\text { Barely } \\
\text { coordinated }\end{array}\right|$ & 0.32 & \begin{tabular}{|l} 
Lagging \\
behind
\end{tabular} & 0.47 & $\begin{array}{l}\text { On the verge of } \\
\text { imbalance }\end{array}$ & 0.53 & $\begin{array}{l}\text { Lagging } \\
\text { behind }\end{array}$ & 0.36 & Mild imbalance & 1.51 & Ahead of & 0.48 & $\begin{array}{l}\text { On the verge } \\
\text { of imbalance }\end{array}$ \\
\hline & 2012 & 0.61 & $\begin{array}{l}\text { Primary } \\
\text { coordination }\end{array} \mid$ & 0.63 & $\begin{array}{l}\text { Lagging } \\
\text { behind }\end{array}$ & 0.58 & $\begin{array}{l}\text { Barely } \\
\text { coordinated }\end{array}$ & 0.78 & $\begin{array}{l}\text { Lagging } \\
\text { behind }\end{array}$ & 0.40 & Mild imbalance & 3.42 & Ahead of & 0.51 & $\begin{array}{l}\text { Barely } \\
\text { coordinated }\end{array}$ \\
\hline & 2013 & 0.66 & $\begin{array}{l}\text { Primary } \\
\text { coordination }\end{array} \mid$ & 0.63 & $\begin{array}{l}\text { Lagging } \\
\text { behind }\end{array}$ & 0.64 & $\begin{array}{l}\text { Primary } \\
\text { coordination }\end{array}$ & 0.69 & $\begin{array}{l}\text { Lagging } \\
\text { behind }\end{array}$ & 0.44 & $\begin{array}{l}\text { On the verge of } \\
\text { imbalance }\end{array}$ & 3.18 & Ahead of & 0.56 & $\begin{array}{l}\text { Barely } \\
\text { coordinated }\end{array}$ \\
\hline & 2014 & 0.71 & $\begin{array}{l}\text { Moderate } \\
\text { coordination }\end{array}$ & 0.77 & $\begin{array}{l}\text { Lagging } \\
\text { behind }\end{array}$ & 0.70 & $\begin{array}{l}\text { Primary } \\
\text { coordination }\end{array}$ & 0.79 & $\begin{array}{l}\text { Lagging } \\
\text { behind }\end{array}$ & 0.52 & $\begin{array}{l}\text { Barely } \\
\text { coordinated }\end{array}$ & 2.70 & Ahead of & 0.62 & $\begin{array}{l}\text { Primary } \\
\text { coordination }\end{array}$ \\
\hline & 2015 & 0.76 & $\begin{array}{l}\text { Moderate } \\
\text { coordination }\end{array}$ & 1.00 & Synchronizing 0 & 0.80 & $\begin{array}{l}\text { Moderate } \\
\text { coordination }\end{array}$ & 0.84 & Synchronizing 0 & 0.62 & $\begin{array}{l}\text { Primary } \\
\text { coordination }\end{array}$ & 2.33 & Ahead of & 0.70 & $\begin{array}{l}\text { Primary } \\
\text { coordination }\end{array}$ \\
\hline & 2016 & 0.88 & $\begin{array}{l}\text { Good } \\
\text { coordination }\end{array}$ & 1.05 & Synchronizing 0 & 0.91 & $\begin{array}{l}\text { High-quality } \\
\text { coordination }\end{array}$ & 0.94 & Synchronizing 0 & 0.80 & $\begin{array}{l}\text { Moderate } \\
\text { coordination }\end{array}$ & 1.54 & Ahead of & 0.85 & $\begin{array}{l}\text { Good } \\
\text { coordination }\end{array}$ \\
\hline & 2017 & 0.96 & \begin{tabular}{|l|} 
High- \\
quality \\
coordination
\end{tabular} & 1.16 & Synchronizing 0 & 0.99 & $\begin{array}{l}\text { High-quality } \\
\text { coordination }\end{array}$ & 1.01 & Synchronizing 0 & 0.88 & $\begin{array}{l}\text { Good } \\
\text { coordination }\end{array}$ & 1.60 & Ahead of & 0.92 & $\begin{array}{l}\text { High-quality } \\
\text { coordination }\end{array}$ \\
\hline \multirow{8}{*}{\begin{tabular}{|c|} 
Beijing- \\
Tianjin- \\
Hebei
\end{tabular}} & 2010 & 0.41 & $\begin{array}{|lr|}\text { On } & \text { the } \\
\text { verge } & \text { of } \\
\text { imbalance }\end{array}$ & 0.27 & $\begin{array}{l}\text { Lagging } \\
\text { behind }\end{array}$ & 0.33 & Mild imbalance & 0.62 & $\begin{array}{l}\text { Lagging } \\
\text { behind }\end{array}$ & 0.45 & $\begin{array}{l}\text { On the verge of } \\
\text { imbalance }\end{array}$ & 0.19 & $\begin{array}{l}\text { Lagging } \\
\text { behind }\end{array}$ & 0.45 & $\begin{array}{l}\text { On the verge } \\
\text { of imbalance }\end{array}$ \\
\hline & 2011 & 0.55 & $\begin{array}{l}\text { Barely } \\
\text { coordinated }\end{array}$ & 0.40 & $\begin{array}{l}\text { Lagging } \\
\text { behind }\end{array}$ & 0.50 & $\begin{array}{l}\text { On the verge of } \\
\text { imbalance }\end{array}$ & 0.63 & $\begin{array}{l}\text { Lagging } \\
\text { behind }\end{array}$ & 0.40 & Mild imbalance & 1.50 & Ahead of & 0.50 & $\begin{array}{l}\text { On the verge } \\
\text { of imbalance }\end{array}$ \\
\hline & 2012 & 0.63 & $\begin{array}{l}\text { Primary } \\
\text { coordination }\end{array}$ & 0.73 & $\begin{array}{l}\text { Lagging } \\
\text { behind }\end{array}$ & 0.62 & $\begin{array}{l}\text { Primary } \\
\text { coordination }\end{array}$ & 0.77 & $\begin{array}{l}\text { Lagging } \\
\text { behind }\end{array}$ & 0.44 & $\begin{array}{l}\text { On the verge of } \\
\text { imbalance }\end{array}$ & 3.05 & Ahead of & 0.54 & $\begin{array}{l}\text { Barely } \\
\text { coordinated }\end{array}$ \\
\hline & 2013 & 0.74 & $\begin{array}{l}\text { Moderate } \\
\text { coordination }\end{array}$ & 1.00 & Synchronizing 0 & 0.75 & $\begin{array}{l}\text { Moderate } \\
\text { coordination }\end{array}$ & 0.94 & Synchronizing 0 & 0.51 & $\begin{array}{l}\text { Barely } \\
\text { coordinated }\end{array}$ & 4.61 & Ahead of & 0.62 & $\begin{array}{l}\text { Primary } \\
\text { coordination }\end{array}$ \\
\hline & 2014 & 0.79 & $\begin{array}{l}\text { Moderate } \\
\text { coordination }\end{array}$ & 1.17 & Synchronizing 0 & 0.81 & $\begin{array}{l}\text { Good } \\
\text { coordination }\end{array}$ & 1.10 & Synchronizing 0 & 0.58 & \begin{tabular}{|l} 
Barely \\
coordinated
\end{tabular} & 4.24 & Ahead of & 0.67 & \begin{tabular}{|l|} 
Primary \\
coordination
\end{tabular} \\
\hline & 2015 & 0.83 & $\begin{array}{l}\text { Good } \\
\text { coordination }\end{array}$ & 1.25 & Ahead of & 0.86 & $\begin{array}{l}\text { Good } \\
\text { coordination }\end{array}$ & 1.10 & Synchronizing 0 & 0.65 & $\begin{array}{l}\text { Primary } \\
\text { coordination }\end{array}$ & 3.36 & Ahead of & 0.73 & $\begin{array}{l}\text { Moderate } \\
\text { coordination }\end{array}$ \\
\hline & 2016 & 0.91 & \begin{tabular}{|l|} 
High- \\
quality \\
coordination
\end{tabular} & 1.15 & Synchronizing 0 & 0.93 & $\begin{array}{l}\text { High-quality } \\
\text { coordination }\end{array}$ & 1.07 & Synchronizing 0 & 0.82 & $\begin{array}{l}\text { Good } \\
\text { coordination }\end{array}$ & 1.75 & Ahead of & 0.86 & $\begin{array}{l}\text { Good } \\
\text { coordination }\end{array}$ \\
\hline & 2017 & 0.94 & \begin{tabular}{|l|} 
High- \\
quality \\
coordination
\end{tabular} & 1.13 & Synchronizing 0 & 0.97 & $\begin{array}{l}\text { High-quality } \\
\text { coordination }\end{array}$ & 1.00 & Synchronizing 0 & 0.88 & $\begin{array}{l}\text { Good } \\
\text { coordination }\end{array}$ & 1.51 & Ahead of & 0.91 & $\begin{array}{l}\text { High-quality } \\
\text { coordination }\end{array}$ \\
\hline
\end{tabular}

In summary, from 2010 to 2017, the coordinated development level of the Beijing-Tianjin-Hebei technology-economy system showed an increasing trend, and the level of coupling coordination transitioned from "on the verge of imbalance" to "highquality coordination". The relative development of the Beijing-Tianjin-Hebei technology-economy system generally went through the "lagging behindSynchronizing" stage. The technological innovation has changed from restricting economic development to promoting economic development, and the system tends to be optimized. From the perspective of the degree of coupling coordination, Beijing and Tianjin have entered the stage of "high-quality coordination" of technology and economy earlier than Hebei.
2) Evaluation of the technology-society coupling coordination development level: According to the established index system, coupling coordination model and relative development model, it substitutes the technology and social development level index into the calculation, and obtains the calculation result of the coordinated development level of technology and society (see "Table III").

It can be seen from "Table III": First, from 2010 to 2017, the development level of the Beijing-TianjinHebei technology-society system coupling coordination showed an increasing trend, the coupling coordination degree rose from 0.33 to 0.97 , and the coupling coordination level transitioned from "mild imbalance" to "high-quality coordination". From the perspective of the trend of the coupling coordination development 
level, the coordinated development of technology and society in Beijing and Tianjin from 2010 to 2014 was faster, and the degree of coupling coordination increased from 0.37 and 0.34 to 0.85 and 0.87 respectively, with an average annual increase of 0.12 and 0.13; Beijing and Tianjin slowed down the coordinated development of technology and society coupling from 2015 to 2017, and the degree of coupling coordination increased from 0.84 and 0.93 to 0.93 and 0.95 respectively, with an average annual growth rate of 0.05 and 0.01 . From 2010 to 2017, Hebei's coordinated development of technology and society was generally faster, and the degree of coupling coordination increased from 0.27 to 0.99 , with an average annual growth rate of 0.1 . From the perspective of the level of coupling coordination development, Tianjin and Hebei have entered the stage of "high-quality coordination" development of technology and society earlier than Beijing.

Second, the relative development of the BeijingTianjin-Hebei technology-society system went through the "lagging behind-synchronization" stage. The technological innovation has changed from restricting social governance to promoting social governance, and the system tends to be optimized. Among them, the relative development relationship between Beijing's technological innovation and social governance is unstable, showing the characteristics of "lagging behind-synchronizing-lagging behind-synchronizingahead of" and "restriction-promotion-restrictionpromotion-influence". Tianjin had been in the stage of lagging technological innovation and restricting social governance from 2010 to 2012. From 2013 to 2017, it was in the stage of synchronizing technological innovation and promoting social governance. Hebei had been in the stage of lagging technological innovation and restricting social governance from 2010 to 2014, and from 2015-2017, it was in the stage of synchronizing technological innovation and promoting social governance.

In summary, from 2010 to 2017, the coordinated development level of Beijing-Tianjin-Hebei technology and society system showed an overall increasing trend, and the level of coupling coordination transitioned from "mild imbalance" to "high-quality coordination". The relative development of the Beijing-Tianjin-Hebei technology-society system went through the "lagging behind-synchronizing" stage and the technological innovation shifted from restricting social governance to promoting social governance, and the system tended to be optimized. From the perspective of the level of coupling coordination development, Tianjin and Hebei have entered the stage of "high-quality coordination" development of technology and society earlier than Beijing.
3) Evaluation of the technology-ecology coupling coordination development level: According to the established index system, coupling coordination model and relative development model, it substitutes the technology and ecological development level index into the calculation, and obtains the calculation result of the coupling coordination development level of technology and ecology (see "Table III").

It can be seen from "Table III": First, from 2010 to 2017, the development level of Beijing-Tianjin-Hebei technology-ecosystem coupling coordination showed a trend of "rising first and then declining". The degree of coupling coordination first dropped from 0.45 to 0.4 and then rose to 0.88 , the level of coupling coordination transitioned from "on the verge of imbalance" to "good coordination". From the perspective of the trend of coupling coordination development level, Beijing, Tianjin and Hebei slowed down the coordinated development of technology and ecological coupling in 2010-2017. The degree of coupling coordination increased from $0.47,0.41,0.36$ to $0.9,0.83$, and 0.88 respectively, with an average annual increase of 0.05 , $0.06,0.07$. From the perspective of the level of coupling coordination development, Beijing and Tianjin entered the stage of "good coordination" of technological and ecological development earlier than Hebei.

Second, from 2010 to 2017, the relative development of technology and ecosystem in BeijingTianjin-Hebei went through a "lagging behind-ahead of" stage. Technological innovation changed from restricting ecological construction to affecting ecological construction, and the system tended to degenerate. Among them, the relative development stages of technology and ecosystem in Beijing, Tianjin, Hebei and Beijing-Tianjin-Hebei were the same, except that Tianjin only showed a "synchronizing" stage in 2012.

In summary, from 2010 to 2017, the coupling coordination development level of the Beijing-TianjinHebei technology-ecosystem showed an overall trend of "rising first and then declining", and the level of coupling coordination changed from "on the verge of imbalance" to "good coordination". The relative development of Beijing-Tianjin-Hebei technology and ecosystem went through a "lagging behind-ahead of" stage. Technological innovation changed from restricting ecological construction to affecting ecological construction, and the system tended to degenerate. From the perspective of the development level of the coupling coordination degree, Beijing and Tianjin entered the stage of "good coordination" of technology and ecology earlier than Hebei. 
4) Evaluation of the technology-economy-societyecology coupling coordination development level: According to the established index system, coupling coordination model and relative development model, it substitutes the scientific and technological, economic, social and ecological development level indexes into the calculation to obtain the evaluation result of the coordinated development of technology-economysociety-ecology coupling (see "Table III") . In addition, due to the coordinated development of the integrated technology-economy-society-ecology coupling within the Beijing-Tianjin-Hebei region is also affected by the technology-ecosystem, technology-economic system,
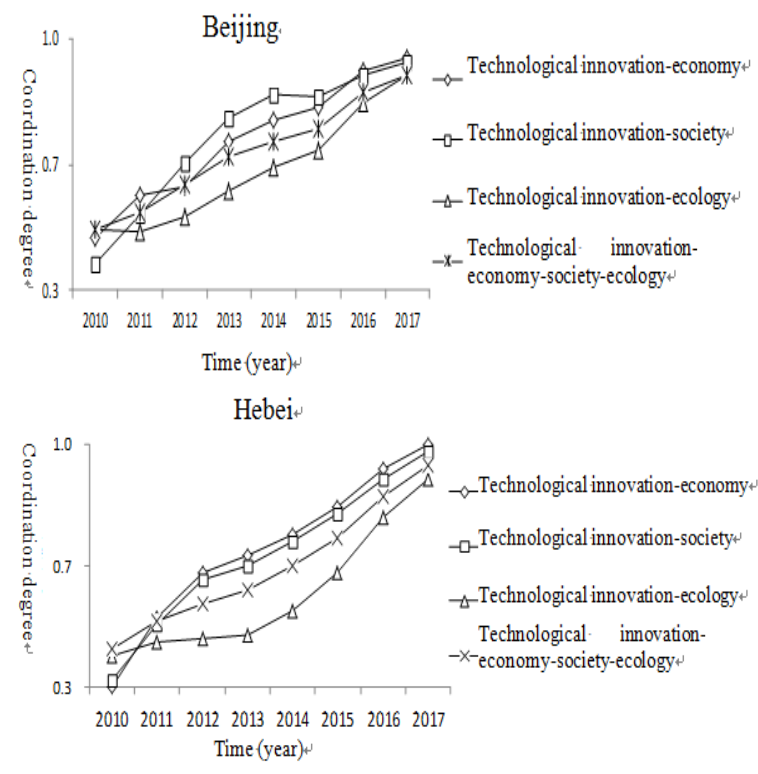

and technology-social system, when the degree of coordination between technology-ecosystem, technology-economic system, and technology-social system is similar, it will promote the overall development of technology-economy-societyecosystem. Conversely, when the degree of coupling coordination differs greatly, it will restrict the overall development of the system. From this, the law of changes in the coordinated development level of technology-economy-society-ecology coupling in the Beijing-Tianjin-Hebei region can be drawn (see "Fig. $1 ")$.

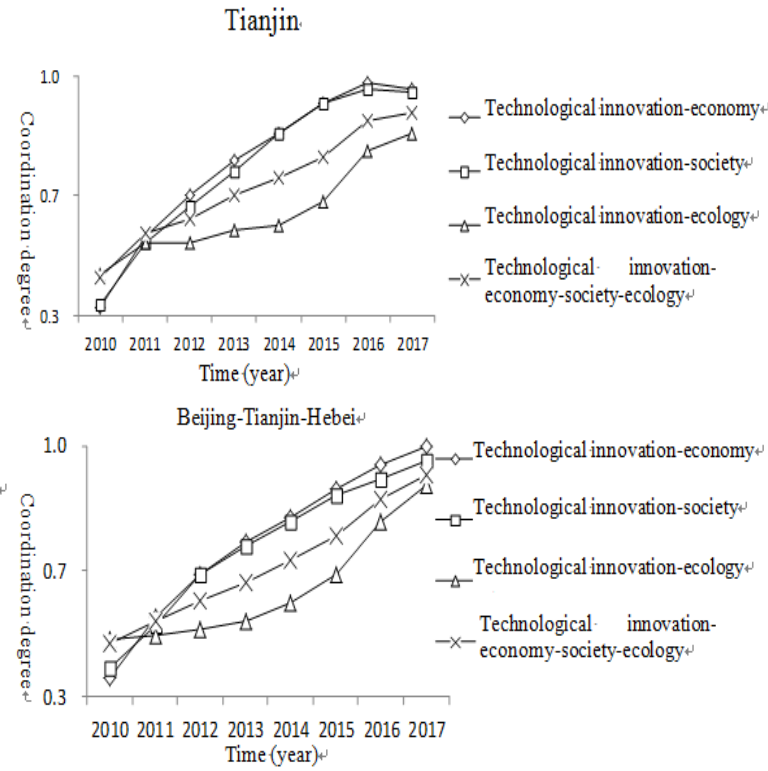

Fig. 1. The Beijing-Tianjin-Hebei region's technology-economy-society-ecology coupling coordination development level.

Combining "Table III" and "Fig. 1", it can be seen that Beijing-Tianjin-Hebei technology-economysociety-ecological overall coupling coordination development level is on the rise, the degree of coupling coordination rises from 0.45 to 0.91 , and the level of coupling coordination changes from "on the verge of imbalance" to "high-quality coordination". From the perspective of the level of coordinated development of technology-economy-society-ecology coupling in the Beijing-Tianjin-Hebei region, from 2010 to 2017, the level of coordinated development of technologyeconomy-society-ecology coupling in Beijing-TianjinHebei was generally higher than that of technologyecosystem and lower than technology -social system and technology-economic system. This shows that the Beijing-Tianjin-Hebei's technology-economic system and the technology-social system have stimulated the coordinated development of the overall coupling of technology-economy-society-ecology, while the technology-ecosystem has restrained its development. By 2017, the coupling coordination development level of Beijing's technology-economy-society-ecology reached 0.9, which was higher than the coordinated development level of technology-ecosystem coupling and was $0.9(0.89)$, and was lower than the technologysocial system and technology-economic system, which were 0.95 and 0.93 respectively; the coupling coordination development level of Tianjin's technologyeconomy-society-ecology reached 0.88 , which was higher than the technology-ecosystem coupling coordination development level and was 0.83 , and lower than the technology-social system and technology-economic system, which were 0.9 and 0.95 respectively; the coupling coordination development level of Hebei's technology-economy-society-ecology reached 0.92 , which was higher than the technologyecosystem coupling coordination development level and was 0.88 , and lower than the technology-social system and technology-economic system, which were 0.96 and 0.99 , respectively. 
To sum up, the overall coupling coordination development level of technology-economy-societyecology in the Beijing-Tianjin-Hebei region is mainly driven by the positive pull of the technology-economic system and the technology-social system as well as the negative inhibition of the technology-ecosystem. The construction of the technology-ecosystem has become a shortcoming that restricts the improvement of the technology-economy-society-ecology coupling coordination ability in the Beijing-Tianjin-Hebei region.

\section{CONCLUSION}

From 2010 to 2017, the Beijing-Tianjin-Hebei region has a significant level of technological, economic and social development, but the level of ecological construction is not good. Among them, in terms of scientific and technological innovation, the development of scientific and technological innovation in Beijing and Hebei is showing a sustained upward trend and the development results are both good. Tianjin's early-stage technological innovation has a strong momentum of development, and its development speed is relatively fast, but its later stage of development has declined slightly. In terms of economic development, Beijing and Hebei continue to show a good trend and the development results are good; Tianjin's economic development momentum is strong, the economic development speed is relatively fast, and the later development level has declined slightly. In terms of social development, Beijing, Tianjin, and Hebei are generally showing a trend of seeking progress while maintaining stability. Beijing's early social development level is relatively good, Tianjin and Hebei develop faster in the later period, but the final result of Hebei's social development is the best. In terms of ecological development, the process and results of ecological construction in Beijing are the best, followed by Hebei, and worst in Tianjin. Under the technology-economic system, the degree of BeijingTianjin-Hebei coupling coordination is increasing, and technological innovation in Beijing-Tianjin-Hebei has changed from restricting economic development to promoting economic development; Beijing and Tianjin have entered the stage of "high-quality coordination" development of technology and economy earlier than Hebei. Under the technology-social system, the degree of Beijing-Tianjin-Hebei coupling coordination is increasing generally, and technological innovation has changed from restricting social governance to promoting social governance; Tianjin and Hebei have entered the stage of "high-quality coordination" development between technology and society earlier than Beijing. Under the technology-ecosystem, the degree of Beijing-Tianjin-Hebei coupling coordination shows a trend of "rising first and then declining", and technological innovation has changed from restricting ecological construction to affecting ecological construction; Beijing and Tianjin have entered the stage of "good coordination" of technology and ecology earlier than Hebei. The overall coupling coordination development of technology-economy-society-ecology in the Beijing-Tianjin-Hebei region is mainly driven by the positive pull of the technology-economic system and the technology-social system as well as the negative inhibition of the technology-ecosystem. That is, the technology-ecology dimension has become a shortcoming that restricts the improvement of the technology-economy-society-ecology coupling coordination ability in the Beijing-Tianjin-Hebei region.

\section{References}

[1] Dong Huizhong, Zhang Feng, Yin Xiuqing. Dynamic correlation analysis of science and technology level and lowcarbon economic development based on extension evaluation [J]. Financial Theory Research, 2015(3):1-8. (in Chinese)

[2] Zhi Huawei, Du Gang, Xie Baichen. Research on the efficiency of regional low-carbon economy from the perspective of science and technology driving $[\mathrm{J}]$. Science and Technology Progress and Countermeasures, 2012, 30(3): 25-31. (in Chinese)

[3] Noda, Hideo. R\&D-Based Models of Economic Growth Reconsidered[J]. INFORMATION-AN INTERNATIONAL INTERDISCIPLINARY JOURNAL,2012,15(2):517-536.

[4] Sun Xijie. Analysis of the exponential law of the coordinated growth of science and technology and the economy - also on the paradox of "exponential growth" in the scale of science and technology [J]. Studies in Science, 2012, 30(6): 813-819. (in Chinese)

[5] Kocsis, Imre,Kiss, Judit T. Renewable energy consumption, R\&D and GDP in European union countries[J]. ENVIRONMENTAL ENGINEERING AND MANAGEMENT JOURNAL, 2014,13(1):2825-2830.

[6] Huang Ying. The relationship between China's investment in science and technology and economic growth: 1978-2009 [J]. Times Finance, 2014(6): 32-33. (in Chinese)

[7] Zhang Youzhi. Science and technology investment and economic growth: a nonlinear relationship with threshold $[\mathrm{J}]$ Forum on Science and Technology in China, 2014(5): 32-38. (in Chinese)

[8] Pan Fanghui, Li Cuixia, Fan Bin. Markov regimes switching model based on the synergy between fiscal science and technology investment cycle and business cycle [J]. Forum on Science and Technology in China, 2015(3): 42-47. (in Chinese)

[9] Beaudreau, Bernard C.,Light foot, H. Douglas. The physical limits to economic growth by R\&D funded innovation [J] ENERGY,2015,84: 45-52.

[10] Moutinho, Ricardo, Au-Yong-Oliveira, Manuel, Coelho, Arnaldo. Beyond the "Innovation's Black-Box": Translating R\&D outlays into employment and economic growth [J]. SOCIO-ECONOMIC PLANNING SCIENCES,2015,50: 45-58.

[11] Kang Shaoda, Wang Jian. Research on the interactive effects of technological progress and urban low-carbon economic development [J]. Macroeconomic Research, 2016(8):116-122. (in Chinese)

[12] Yang Wu, Yang Miao. Coupling degree model of China's science and technology innovation and economic development [J]. Forum on Science and Technology in China, 2016(3): 3035. (in Chinese) 
[13] Gu Weinan, Shen Yuming, Wang Shuhua, et al. The spatial evolution of scientific and technological innovation capabilities and its relationship with economic development [J]. Forum on Science and Technology in China, 2017(9): 23-29. (in Chinese)

[14] Xiang Li. The temporal and spatial characteristics of the coordinated development of scientific and technological innovation and ecological environment in China's provinces [J]. Technological Economy, 2016, 35(11): 28-35. (in Chinese)

[15] Li Dongdong, Xu Zhengliang, Zhou Huiguang. Evaluation of the impact of scientific and technological investment on regional ecological environment optimization [J]. Modern Management Science, 2013(5): 63-65+71. (in Chinese)

[16] Han Bin, Kong Jijun. Rethinking on technological innovation and low-carbon development in Yunnan [J]. China Population, Resources and Environment, 2014, 24(3): 24-26. (in Chinese)

[17] Sun Jianjing. Research on the coordinated development model of technology, economy and society [J]. Chinese Management Science, 1996(2): 13-18. (in Chinese)

[18] Guo Jiangjiang, Qi Wei, Miao Yajun. Research on the measurement of the coordination of China's science and technology and economic society development $[\mathrm{J}]$. Forum on Science and Technology in China, 2012(5): 123-129. (in Chinese)

[19] Wang Wei, Zhang Jianye, Qiao Penghua. Research on the coordinated development of regional scientific and technological talents, industrial economy and ecological environment - Based on panel data of 18 larger cities in China [J]. Science and Technology Progress and Countermeasures, 2014, 31(7): 37-42. (in Chinese)

[20] Wu Dan, Hu Jing. Evaluation of the comprehensive development level and coordination degree of China's technology-economy-ecosystem - based on the gray relation projection pursuit coordination degree combination evaluation model [J]. Industrial Technology Economy, 2017, 35( 5): 140146. (in Chinese)

[21] Wen Xianming, Wang Ce, Xiong Ying, et al. Coupling coordination development of new urbanization and financial support in Hunan Province [J]. Economic Geography, 2019,39(07):96-105. (in Chinese) 